\title{
NEAREST-NEIGHBOR DISTANCES IN DIFFUSION-CONTROLLED REACTIONS MODELLED BY A SINGLE MOBILE TRAP
}

\author{
Rodney SCHOONOVER ${ }^{1}$, Daniel BEN-AVRAHAM ${ }^{2}$, \\ Shlomo HAVLIN ${ }^{3.4}$, Raoul KOPELMAN ${ }^{1}$ and George H. WEISS ${ }^{3}$ \\ 'Department of Chemistry, University of Michigan, Ann Arbor, MI 48109, USA \\ ${ }^{2}$ Department of Physics, Clarkson University, Potsdam, NY 13676, USA \\ ${ }^{3}$ National Institutes of Health, Bethesda, MD 20892, USA \\ ${ }^{4}$ Permanent address: Department of Physics, Bar-Ilan University, 52100 Ramat-Gan, Israel
}

\section{Received 13 April 1990}

Revised manuscript received 26 June 1990

\begin{abstract}
We consider a system consisting of an infinite number of identical particles on a lattice initially uniformly distributed. which diffuse in the presence of a single mobile trap and ask for the time-dependent behavior of the distance of the trap from the nearest particle. This quantity is a measure of the tendency of the system to self-segregate. We show, by a simulation incorporating the exact enumeration method, that in one dimension the expected distance $\langle L(t))$ scales as $\langle L(t)\rangle \sim t^{\alpha}$ as $t \rightarrow \infty$, where the exponent $\alpha$ depends only on the ratio of the diffusion constants. A heuristic expression for $\alpha$ is suggested, analogous to a rigorous exponent found by ben-Avraham for a similar but not identical problem. The flux into the trap is found to vary as $t^{-1 / 2}$ independent of the diffusion constants
\end{abstract}

\section{Introduction}

Widespread application of the Smoluchowski theory of reaction rates in the literature of chemical physics [1] suggests both the interest and importance of examining some of the physical effects omitted in the analysis of this grossly simplified model. In particular, one would like to gain some motion of whether, and by how much, many-body effects are likely to change the Smoluchowski model. In general this poses a formidable problem and only partial and approximate results are available, which are mainly due to Waite $[2,3]$. More recently attention has been focussed on simplified one-dimensional systems exemplified by the reaction $\mathrm{A}+\mathrm{B} \rightarrow \mathrm{B}$, which is clearly an easier version of the original three-dimensional Smoluchowski model [4].

In the Smoluchowski model one starts from a picture consisting of a single B molecule that remains fixed in space while an infinite number of $A$ particles, initially uniformly distributed throughout space, perform Brownian motion 
without mutual interaction. A reaction is said to occur when an A comes into contact with the $B$. In the original, as well as in most later formulations of the Smoluchowski model, the key assumption that considerably simplifies the analysis is that the A particles, which are idealized as geometric points, diffuse without interacting with one another. Recently there has been considerable interest in the behavior of reacting systems in low dimensions [5-12], where the classical reaction laws require modification due to effects exemplified by the self-segregation of reactants. One of the conceptually simplest generalizations of the Smoluchowski model is one in which both $A$ and $B$ particles are allowed to diffuse, with possibly different diffusion constants $D_{A}$ and $D_{B}$. A significant feature of such a model is that the diffusion of both species induces correlations in the distance from the $B$ to individual A's, even in the absence of any physical interaction between the A's. Such correlations are most evident in low dimensions, and play a much less significant role in determining the reaction kinetics of three-dimensional systems. A mathematical analysis of even the most simplified version of the modified Smoluchowski model appears to be intractable, and to date there is only one approximate snalysis of the kinetics of such a model in the literature [13].

One quantitative measure of the tendency of low-dimen: ional reacting systems to self-segregate is the distance of the $B$ particle fre $n$ the nearest unreacted $A$, i.e., the generalization of the Hertz distribution $[i, A]$ that is valid when neither A's nor the B can move. Analyses of this quantity have appeared in the limiting cases in which the $B$ is stationary [15] and the A's diffuse. and in the contrary case in which the $B$ diffuses while the A's are stationary [16]. The difference between these two cases can be characterized in terms of the mean value of the distance of $B$ from the closest $A$, and is most striking in the case of a one-dimensional system. Let $\langle L(t)\rangle$ denote the mean nearest-neighbor distance at time $t$. It has been shown that for the stationary-B mobile-A model in one dimension [15]

$$
\lim _{t \rightarrow \infty} \frac{\langle L(t)\rangle}{t^{1 / 4}}=\text { constant }
$$

while in the mobile-B stationary-A model one has [16]

$$
\lim _{t \rightarrow \infty} \frac{\langle L(t)\rangle}{t^{1 / 2}}=\text { constant }
$$

In the present note we investigate, by a form of simulation based in part on the exact enumeration method [17], the asymptotic behavior of $\langle L(t)\rangle$ for cases intermediate between those just mentioned, in which both A's and B's are allowed to diffuse. 


\section{Results}

Our simulations were performed for a discrete nearest-neighbor random walk model of the diffusion process in one dimension in which, on any given step, the probability that a particle moves from site $i$ to $i \pm 1$ is taken equal to $\epsilon / 2$ and the probability of making no step is equal to $1-\epsilon$. The corresponding diffusion constant in a continuous model is then proportional to $\epsilon$. In the following presentation and discussion of results we will refer to $\epsilon$ as the diffusion constant. In our simulation the motion of the B particle was prescribed by a Monte Carlo method while the effects of motion of the more numerous A particles was handled by the exact enumeration method [17]. This has proved to yield highly accurate results in applications where exact results can be used to check the simulations (cf., for example, ref. [18]). In all cases we started with a semi-infinite lattice consisting of 2000 sites. The probability that a given site initially contains an $\mathrm{A}$ was set equal to 0.8 at $t=0$, this high concentration being chosen in order to minimize sampling errors insofar as possible. Initially the B particle is set at one end of the line, but, since it is allowed to move either to the right or to the left, the line can be greater than its original 2000 sites after a number of steps of the run. For each value of the parameters we made runs based on 500 initial A configurations (except for the case in which $D_{\mathrm{A}}=D_{\mathrm{B}}$, which is based on 5000 initial A configurations) averaging over the results to draw a $\log -\log$ plot, which allows us to estimate the exponent $\alpha$ in the conjectured relation

$$
\lim _{t \rightarrow x} \frac{\langle L(t)\rangle}{t^{\alpha}}=\text { constant }
$$

generalizing eqs. (1) and (2).

Some typical plots of $\ln \langle L(t)\rangle$ against $\ln t$ are shown in fig. 1, together with the best-fit straight line through the data. Other cases not shown in the figure lead to equally convincing straight lines. We have also compared data obtained with different values of $D_{\mathrm{A}}$ and $D_{\mathrm{B}}$ for fixed values of the ratio $D_{\mathrm{B}} / D_{\mathrm{A}}$ finding that the values of the exponent $\alpha$ remains the same to within the sampling error. As an example of the results obtained, the exponent calculated using $D_{\mathrm{A}}=D_{\mathrm{B}}=1$ yields an exponent equal to 0.319 . The corresponding exponent for $D_{A}=D_{B}=0.5$ was found to equal 0.314 . The $99 \%$ confidence intervals associated with these exponents, on the assumption that errurs have a Gaussian distribution [20], were calculated to be $(0.307,0.330)$, and $(0.296,0.331)$ respectively, for the two sets of data. For the sake of comparison we also find that in the case $D_{\mathrm{B}}=1$ and $D_{\mathrm{A}}=0$ the $99 \%$ confidence interval for our data is $(0.449,0.488)$. We also varied the sets $\left(D_{\mathrm{A}}, D_{\mathrm{B}}\right)$ corresponding to other fixed 


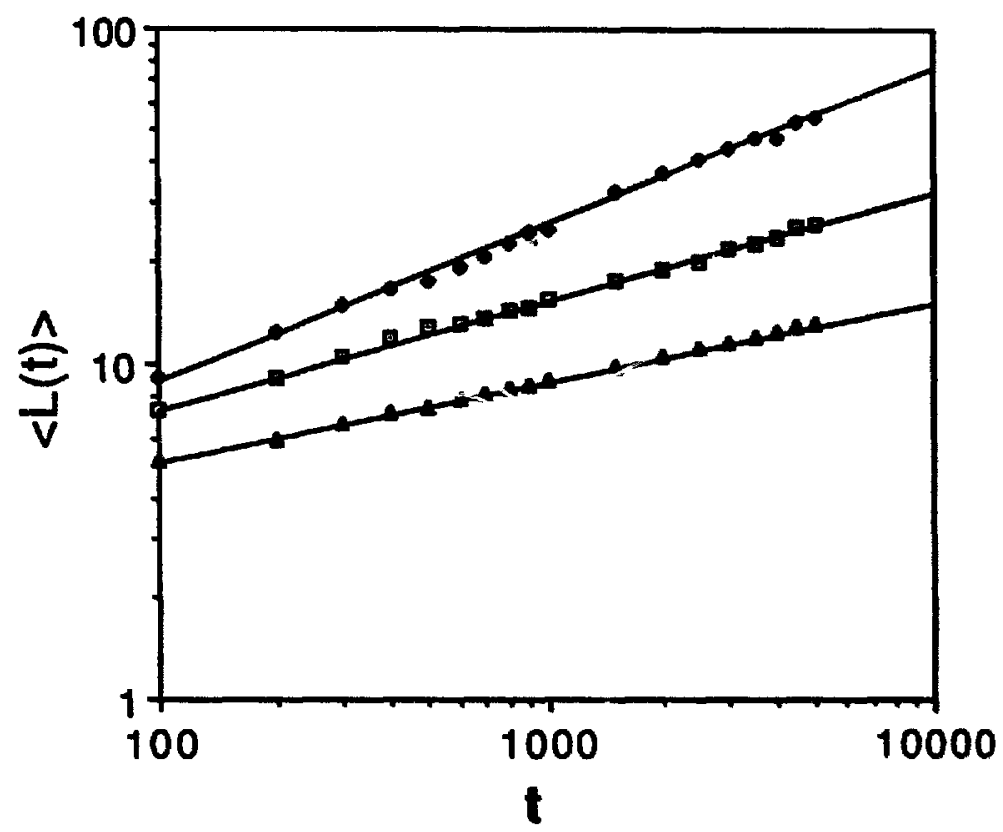

Fig. 1. Data for $\langle L(t)\rangle$ as a function of $t$ on a $\log$-log plot. together with the approximating straight lines. The three lines represent: $(\bullet) D_{A}=0, D_{B}=1 ;(\square) D_{A}=1, D_{B}=1 ;(A) D_{A}=1$. $D_{\mathrm{B}}=\mathbf{0}$.

values of $D_{\mathrm{B}} / D_{\mathrm{A}}$, finding no significant differences in the exponents. This suggests that the exponent $\alpha$ indeed depends only on the ratio. and not separately on the two diffusion constants.

The exponent calculated by simulation for the case $D_{\mathrm{A}}=0, D_{\mathrm{B}} \neq 0$ is estimated to be 0.469 , which should be compared with the theoretical value of 0.5 , while the exponent for $D_{\mathrm{A}} \neq 0, D_{\mathrm{B}}=0$ is estimated to be 0.247 , to be compared to the theoretical value of 0.25 . An additional set of simulations was generated in which the concentration of A particles is kept fixed at 1 at the edges of the lattice to mimic the situation in which the line is actually infinite. The results obtained in this way yielded results in excellent agreement with our first set. While we have no theory to predict the value of $\alpha$ in terms of the diffusion constants, it is possible to conjecture a form for this parameter, based on an analogy with the exponent found by ben-Avraham [19], in an analysis of the double-diffusion problem in which there is a single B particle surrounded by two A particles. The exponent in ref. [19] appears in an expression for the survival time. While the extrapolation from a three-particle system to an infinite particie system is a considerabie one, based on it, we suggest that $\alpha$ should be of the form

$$
\alpha=\frac{1}{\pi} \tan ^{-1} \sqrt{1+2 \frac{D_{\mathrm{B}}}{D_{\mathrm{A}}}} .
$$

This correctly yields the results at the extreme values, $D_{\mathrm{B}} / D_{\mathrm{A}}=0$ and $\propto$. A 
comparison of values of $\alpha$ obtained by simulation and those found from the conjectured value in eq. (4) is given in table $I$. The values have been calculated using one set of values of the pair $\left(D_{\mathrm{A}}, D_{\mathrm{B}}\right)$ for each ratio $D_{\mathrm{B}} / D_{\mathrm{A}}$. The reader will observe that values of $\alpha$ calculated from eq. (4) are consistently higher than our estimated values. Two possibilities can be put forward to account for the observed discrepancies. Either the simulations have not been carried out to large enough times or else eq. (4) should be regarded as an approximation and not an exact result. Possible evidence for the first possibility is contained in the fact that the simulation results are low in the two cases $(0$ and $\infty)$ in which $\alpha$ is known exactly.

Finally, we propose a possible form for the asymptotic form of the probability density, $f(L, t)$, for the nearest-neighbor distance $L(t)$. This is a scaling form in which time enters only through $\langle L(t)\rangle$, i.e.,

$$
f(L, t)=\frac{N}{\langle L(t)\rangle} g\left(\frac{L}{\langle L(t)\rangle}\right),
$$

where $g(x)$ is to be found from our simulations and $N$ is the normalizing constant

$$
N=1 / \int_{0}^{x} g(x) \mathrm{d} x .
$$

The asymptotic forms for $f(L, t)$ when $D_{\mathrm{B}} / D_{\mathrm{A}}=0$ and $\propto$ have the scaling form suggested in eq. (5). In fig. 2 we present a histogram for the function $g(x)$ based on data for $t=1000$ and 5000 (in discrete time) plotted as a function of $L /\langle L(t)\rangle$ for $D_{\mathrm{B}} / D_{\mathrm{A}}=1$. The approximate agreement between this scaling

Table I

Comparison of the estimated value of $\alpha$ with the conjectured prediction of eq. (4).

\begin{tabular}{cll}
\hline$D_{\mathrm{B}} / D_{\mathrm{A}}$ & $\alpha$ from simulations & $\alpha$ from eq. (4) \\
\hline 0.0 & 0247 & 0250 \\
0.1 & 0.259 & 0.264 \\
0.2 & 0.270 & 0277 \\
0.5 & 0.290 & 0304 \\
1.0 & 0.319 & 0333 \\
2.0 & 0.350 & 0366 \\
4.0 & 0.380 & 0398 \\
5.0 & 0390 & 0407 \\
10.0 & 0.410 & 0.432 \\
$x$ & 0.469 & 0500 \\
\hline
\end{tabular}




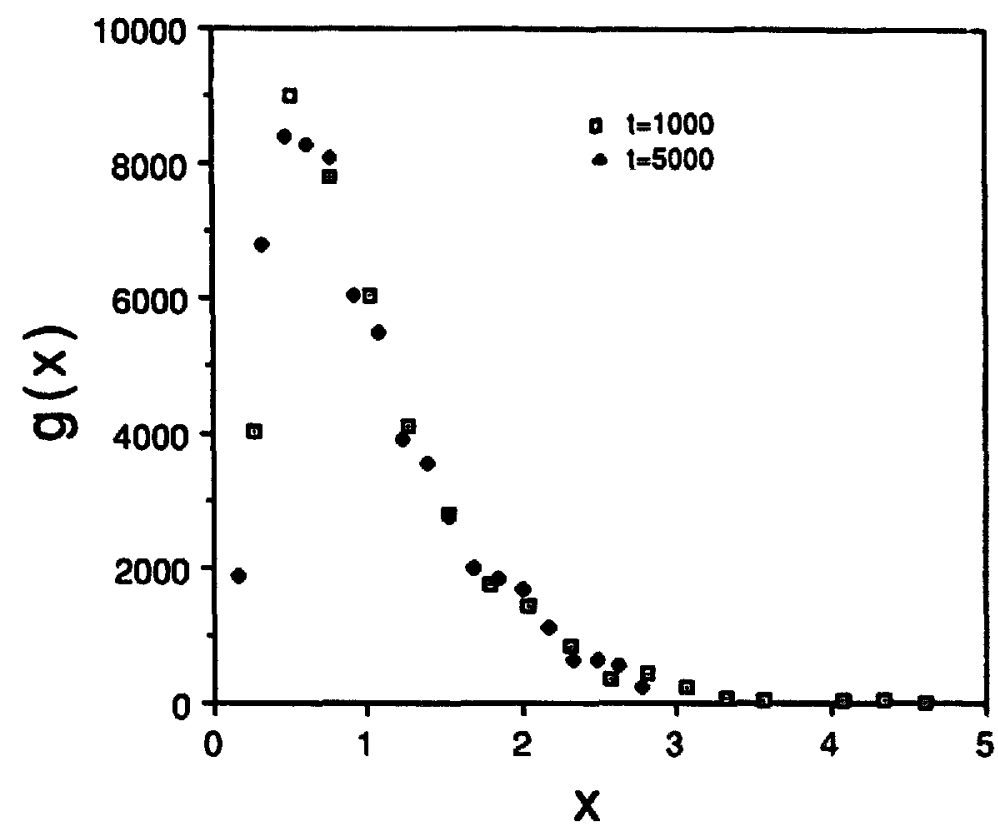

Fig. 2. A histogram for approximation for the function $g(x)$ at times $t=1000$ and 5000 and $D_{\mathrm{A}}=D_{\mathrm{B}}=1$. The collapse onto a single scaled curve is evident.

form and our data is evident from the figure. A good fit to the resulting curve is found by using the skewed-exponential form

$$
g(x)=x^{1.32} \exp (-2.64 x) \text {. }
$$

One further quantity of interest in characerizing the kinetic behavior of the system is the reaction rate, or flux at the trap. Fig. 3 shows the results of runs made with several sets of diffusion constants. The apparent straight lines on the $\log -\log$ plots all have slopes very close to $-1 / 2$ which is the result found for the particular cases treated in earlier investigations $[15,16]$.

While most of our conclusions are based on the exact enumeration technique, we have also performed a large number of numerical experiments in which the diffusion process was simulated, i.e., a pure Monte Carlo technique. In all cases the results of both approaches were in good agreement.

\section{Acknowledgements}

The work of R.S. and R.K. was supported by NSF Grant No. DMR 8801120. D. b-A is grateful for the support of a grant from the Petroleum Research Foundation. The work of S.H. was partially supported by the U.S.-Israel Bi-National Science Foundation. 


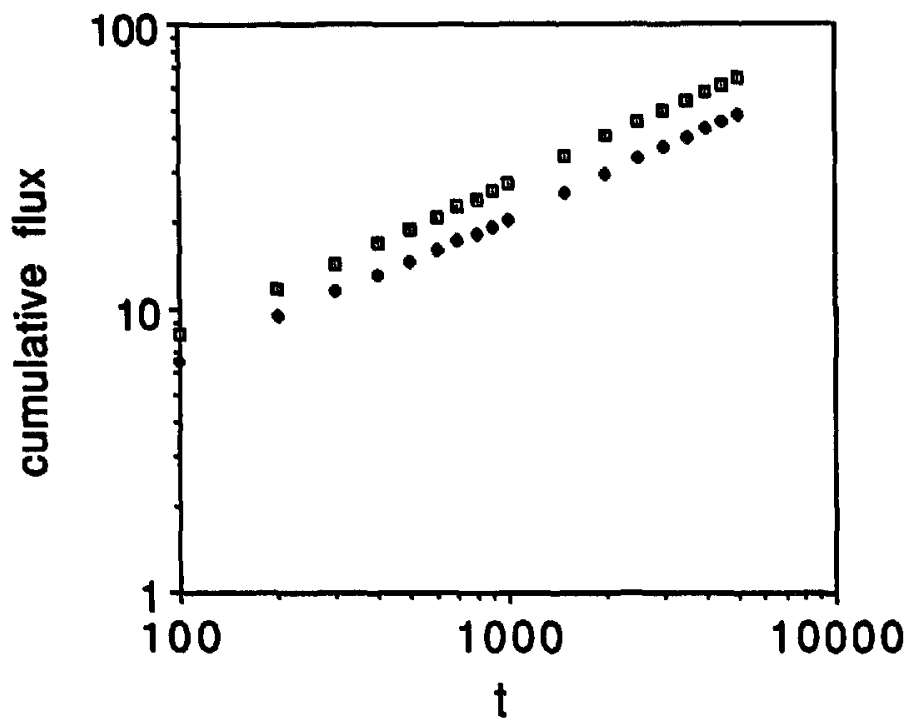

Fig. 3. Data for the cumulative flux at the trap as a function of step number on a log-log plot for: () $D_{\mathrm{A}}=1, D_{\mathrm{B}}=1 ;(\mathrm{A}) D_{\mathrm{A}}=1, D_{\mathrm{B}}=0.2$. The two lines have slopes very close to $1 / 2$. Other data sets not included in the figure show the same behavior.

\section{References}

[1] S.A. Rice, Diffusion-Limited Reactions (Elsevier, Amsterdam, 1985)

[2] T.R. Waite, Phys. Rev. 107 (1957) 463, 471.

[3] T.R. Waite, J. Chem. Phys. 28 (1958) 103.

[4] M.v. Smoluchowski, Z Phys. Chem. 92 (1917) 129

[5] A.A. Ovchinnikov and Y.B. Zeldovich, Chem. Phys. 28 (1978) 215.

[6] D. Toussaint and F. Wilczek, J. Chem. Phys. 78 (1983) 2642.

[7] L.W Anacker and R. Kopelman, Phys. Rev. Lett 58 (1987) 289.

[8] J.S Newhouse and R. Kopelman, J. Phys. Chem 92 (1988) 1538.

[9] R. Kopelman, Science 241 (1988) 1620.

[10] V. Kuzovkov and E. Kotomin, Rep. Prog. Phys. 51 (1988) 1479.

[11] K. Lindenberg, B.J. West and R. Kopelman, Phys. Rev. Lett. 60 (1988) 1777.

[12] S.J. Parus, P. Prasad and R. Kopelman, Chem. Phys. 209 (1988) 128.

[13] A. Szabo, R. Zwanzig and N. Agmon, Phys. Rev. Lett. 61 (1988) 2496.

[14] S. Chandrasekhar, Rev. Mod. Phys. 15 (1943) 1.

[15] G.H. Weiss, R. Kopelman and S. Havlin, Phys. Rev. A 39 (1989) 466.

[16] D. ben-Avraham and G.H. Weiss, Phys. Rev. A 39 (1989) 6436.

[17] S. Havlin and D. ben-Avraham, Adv. Phys. 36 (1987) 695.

[18] S. Havlin, G.H. Weiss, J.E. Kiefer and M. Dishon, J. Phys. A 17 (1984) L347.

[19] D. ben-Avraham, J. Chem. Phys. 88 (1988) 941.

[20] D.C. Montgomery and E.A. Peck, Introduction to Linear Regression Analysis (Wiley, New York, 1982). 